\title{
Spectral tuning of plasmon-enhanced silicon quantum dot luminescence
}

\author{
Julie S. Biteen, ${ }^{\text {a) }}$ Nathan S. Lewis, and Harry A. Atwater \\ California Institute of Technology, 1200 East California Boulevard, Pasadena, California 91125 \\ Hans Mertens and Albert Polman \\ Center for Nanophotonics, FOM Institute for Atomic and Molecular Physics, Kruislaan 407, \\ 1098 SJ Amsterdam, The Netherlands
}

(Received 9 November 2005; accepted 16 February 2006; published online 31 March 2006)

\begin{abstract}
In the presence of nanoscale silver island arrays, silicon quantum dots exhibit up to sevenfold luminescence enhancements at emission frequencies that correspond to the collective dipole plasmon resonance frequency of the Ag island array. Using electron-beam lithography to alter the pitch and particle diameter, this wavelength-selective enhancement can be varied as the metal array resonance wavelength is tuned from 600 to $900 \mathrm{~nm}$. The luminescence intensity enhancement upon coupling is attributed to an increase in the radiative decay rate of the silicon quantum dots. (C) 2006 American Institute of Physics. [DOI: 10.1063/1.2191411]
\end{abstract}

Since the observation in 1990 of strong roomtemperature photoluminescence from porous silicon, ${ }^{1,2}$ significant worldwide interest has been directed toward siliconbased photonics for integrated optoelectronics. An integral part of such systems is a Si-based, power-efficient light emitter. From that perspective, silicon quantum dots (Si QDs) have been intensively investigated as light sources. Si QDs exhibit efficient room-temperature luminescence due to the radiative recombination of quantum-confined excitons, and their emission frequency can be tuned through part of the visible and near-infrared spectrum by varying the QD size ${ }^{3}$ or surface termination. ${ }^{4}$ However, Si QDs suffer from low radiative decay rates relative to those of direct band gap semiconductors and organic dyes. Coupling of radiative dipole emission to metal nanostructures provides an opportunity to enhance the Si QD radiative decay rate, as previous reports have shown for semiconductor QDs (Refs. 5-7) and quantum wells. ${ }^{8}$ Enhanced radiative emission has been attributed to electromagnetic coupling between the semiconductor active dipole emitters and surface plasmons in the metal. This coupling, which is stronger at frequencies near the plasmon resonance, can result in enhancements of the emission intensity and of the decay rates. ${ }^{9}$

In this letter, we report an enhancement of photoluminescence (PL) in Si QDs coupled to Ag island arrays fabricated by electron-beam lithography. When the surface plasmon resonance of the $\mathrm{Ag}$ island arrays is tuned throughout the Si QD emission spectrum, we observe a strong correlation between the frequency at which the PL emission is enhanced and the surface plasmon resonance frequency. From this behavior, we conclude that the observed PL enhancement is caused by an enhancement of the radiative decay rate of the Si QDs.

$\mathrm{Si}$ QDs were produced by ion implantation of $11-\mathrm{keV}$ $\mathrm{Si}^{+}$ions to a fluence of $1.7 \times 10^{16} \mathrm{~cm}^{-2}$ into a 1.6 -mm-thick fused quartz strip (Technical Glass Products). Monte Carlo simulations performed with SRIM (Ref. 10) indicate that such an implantation yields a Gaussian depth distribution of Si in the $\mathrm{SiO}_{2}$, with a peak excess $\mathrm{Si}$ concentration of $10 \%$ at a depth of $\sim 20 \mathrm{~nm}$. The implanted quartz was annealed in $\mathrm{Ar}$

\footnotetext{
a) Author to whom correspondence should be addressed; electronic mail: jsbiteen@caltech.edu
}

for $20 \mathrm{~min}$ each at 200 and $450{ }^{\circ} \mathrm{C}$, and then for $30 \mathrm{~min}$ at $1000{ }^{\circ} \mathrm{C}$, to form $\mathrm{Si}$ QDs with typical diameters of $3-5 \mathrm{~nm} .{ }^{11}$ The samples were subsequently heated in forming gas $\left(10 \% \mathrm{H}_{2}, 90 \% \mathrm{~N}_{2}\right)$ for $30 \mathrm{~min}$ at $450{ }^{\circ} \mathrm{C}$ to eliminate emission from defect states in the $\mathrm{SiO}_{2}$ matrix. ${ }^{12}$ After Si QD formation, the QD distribution was brought to an average depth $\Delta$ of 8,13 , or $18 \mathrm{~nm}$ from the surface by etching in $2.3 \% \mathrm{HF}(\mathrm{aq})$. The etch rate was $0.25 \mathrm{~nm} / \mathrm{s}$, as determined by spectroscopic ellipsometry (Sentech SE-850) on an analogous sample of $\mathrm{SiO}_{2}$ on a $\mathrm{Si}$ substrate. Ellipsometry also indicated that after etching, the surface roughness was less than $\pm 0.2 \mathrm{~nm}$.

To obtain the strongest possible plasmonic effects, the $\mathrm{Si}$-QD-doped $\mathrm{SiO}_{2}$ was coupled to $\mathrm{Ag}$ nanoparticles. Silver was chosen because it has the lowest absorption and produces the strongest resonances among all metals with plasmon resonances at visible frequencies. The surface of the QD-doped quartz was cleaned in a 5:1:1 $\mathrm{H}_{2} \mathrm{O}: \mathrm{H}_{2} \mathrm{O}_{2}: \mathrm{NH}_{4} \mathrm{OH}$ solution. An electron beam was used to pattern $100 \mu \mathrm{m} \times 100 \mu \mathrm{m}$ arrays of circles on two layers of polymethylmethacrylate (PMMA) covered by a $17 \mathrm{~nm} \mathrm{Ge}$ conduction layer. After removing the Ge and developing the resist, a $2 \mathrm{~nm} \mathrm{Si} \mathrm{wetting} \mathrm{layer} \mathrm{and} \mathrm{a} 20 \mathrm{~nm} \mathrm{Ag}$ layer were deposited onto the structure by electron-beam evaporation under ultrahigh vacuum conditions. The remaining PMMA was lifted off, leaving arrays of cylindrical Ag islands. The resulting structure is sketched in Fig. 1(a), and scanning electron micrograph (SEM) images of one series of $\mathrm{Ag}$ arrays, with a $400 \mathrm{~nm}$ pitch and increasing diameters (135-390 nm), are displayed in Fig. 1(b).

Each array of Ag islands with height $h=20 \mathrm{~nm}$ was characterized by a distinct pitch $p(200-500 \mathrm{~nm})$, particle diameter $d(100-440 \mathrm{~nm})$, and thus cylinder aspect ratio $a=d / h(5-22)$. As a consequence of these geometric differences, the arrays were characterized by distinct plasmon resonance frequencies, ${ }^{13}$ which were determined by measuring the transmission spectrum through each Ag island array. For the transmission measurement, the arrays were illuminated with a white light source incident through a $60 \times$ microscope objective, and the transmitted light was collected with a $20 \times$ microscope objective. The spectral transmittance through each Ag island array was normalized by the transmittance through a nearby region devoid of $\mathrm{Ag}$ islands. 

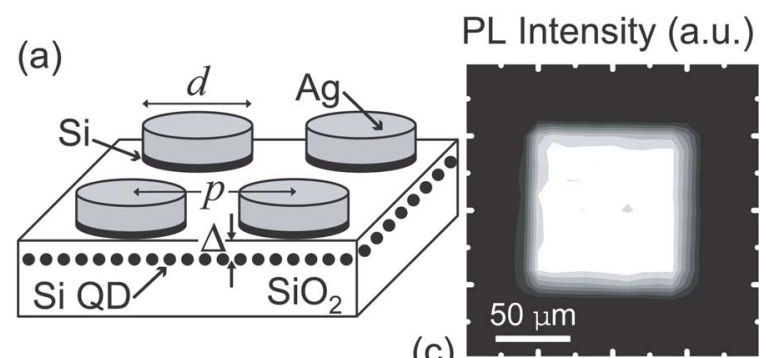

(b)
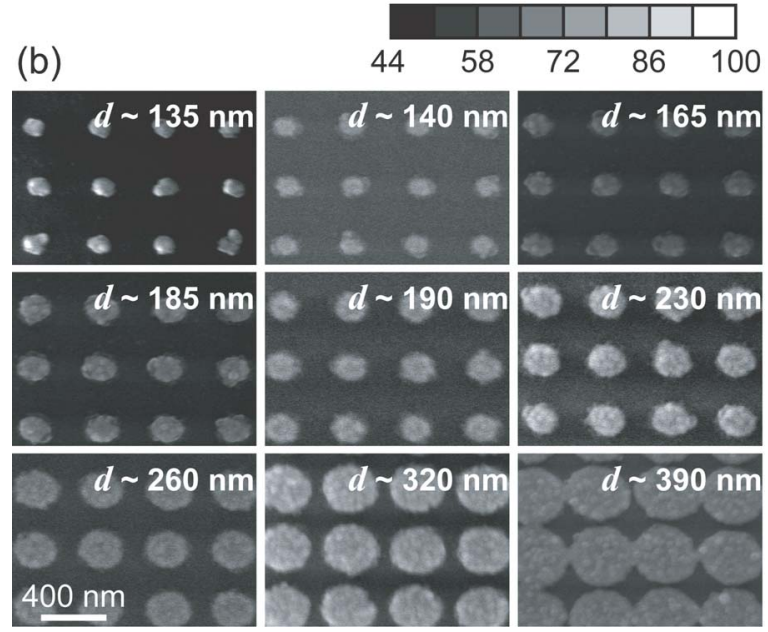

FIG. 1. (a) Array of Ag nanoparticles with diameter $d$ and pitch $p$ on SiQD-doped quartz. (b) SEM images of a series of Ag nanoparticle arrays with $p=400 \mathrm{~nm}$ and $d=135-390 \mathrm{~nm}$. (c) PL intensity map over a representative Ag array $(p=400 \mathrm{~nm}, d=320 \mathrm{~nm}$, and $\Delta=8 \mathrm{~nm})$ in $10-\mu \mathrm{m}$ steps, integrated from $\lambda=640$ to $950 \mathrm{~nm}$.

PL spectra were acquired under excitation from a frequency-doubled Nd:YAG (yttrium aluminum garnet) laser operating at $\lambda_{\text {excitation }}=532 \mathrm{~nm}$ focused through a microscope objective to a $10-\mu \mathrm{m}$-diam spot. A relatively high pump power, $10^{4} \mathrm{~W} / \mathrm{cm}^{2}$, was used to ensure operation near the saturated pump power regime, where the PL emission is limited by the radiative decay rate and is independent of the quantum efficiency. The PL intensity was recorded using a thermoelectrically cooled charge-coupled device detector (sensitivity range of $200-1100 \mathrm{~nm}$ ) in conjunction with a $30 \mathrm{~cm}$ focal length grating spectrograph. A dichroic filter that absorbs wavelengths below $550 \mathrm{~nm}$ was used to eliminate incident laser light from the detector. Si QD excitation and PL emission collection were performed both through the Ag arrays and through the quartz substrate, and all of these combinations gave similar results for the enhancement magnitude and spectral dependence, indicating that the $\mathrm{Si} / \mathrm{Ag}$ system was acting as a coupled entity. Reference luminescence measurements were obtained from a region of Si-QD-doped quartz not located under a Ag island array. To check for PL signal uniformity over the range of the $100 \mu \mathrm{m} \times 100 \mu \mathrm{m}$ particle arrays, as well as over the range of the Ag-free reference areas, the samples were mounted on a translation stage and the PL intensity was measured in $10-\mu \mathrm{m}$ steps. Figure 1(c) shows a typical result for such a two-dimensional scan $(d=320 \mathrm{~nm}, p=400 \mathrm{~nm}$, and $\Delta=8 \mathrm{~nm})$. The PL intensity (integrated from $\lambda=640$ to $950 \mathrm{~nm}$ ) emitted from the array region was approximately twice that emitted from the uncoupled QDs in the reference areas, and the PL intensity was essentially constant in both areas. The spatial resolution of this measurement is not sufficient to identify differences Downloaded 24 May 2006 to 192.87.154.163. Redistribution subject

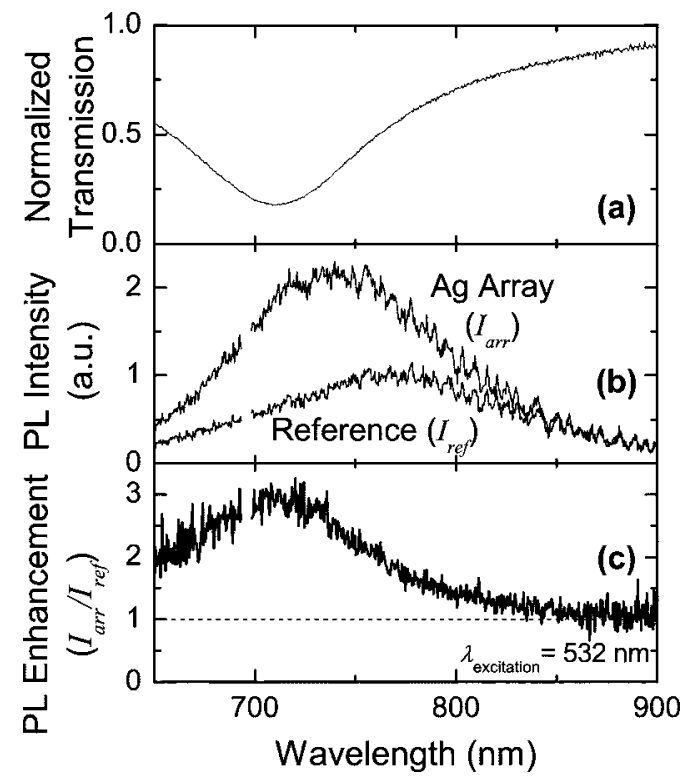

FIG. 2. Optical characterization of a representative sample ( $p=400 \mathrm{~nm}$, $d=320 \mathrm{~nm}$, and $\Delta=13 \mathrm{~nm}$ ). (a) Normalized transmission through the $\mathrm{Ag}$ nanoparticle array. (b) PL intensity from Si QDs in the absence (bottom curve) and presence (top curve) of Ag islands. (c) PL intensity enhancement [ratio of curves in (b)].

in PL intensity at length scales smaller than the pitch.

Figure 2 shows the correspondence between the spectral position and shape of the Si QD PL enhancement and the surface plasmon resonance of a typical Ag island array/Si QD system $(d=190 \mathrm{~nm}, p=400 \mathrm{~nm}$, and $\Delta=13 \mathrm{~nm})$. The normalized transmission spectrum of the array [Fig. 2(a)] exhibited a dip centered at $710 \mathrm{~nm}$ that can be attributed to extinction due to the excitation of dipole plasmon modes in the Ag islands. As illustrated in Fig. 2(b), the PL intensity on the array $I_{\text {arr }}$ was enhanced significantly compared to the $\mathrm{Si}$ QD PL intensity in the reference area $I_{\text {ref }}$, especially at wavelengths where the plasmon mode is resonant. Figure 2(c) shows the PL enhancement spectrum, $I_{\text {arr }}(\lambda) / I_{\text {ref }}(\lambda)$. The enhancement spectrum corresponds strongly with the transmission spectrum, peaking at $\lambda=710 \mathrm{~nm}$.

Figure 3 shows normalized transmission and PL enhancement spectra for six samples with $\mathrm{Ag}$ island arrays that have an increasing island diameter from top to bottom at a fixed $400 \mathrm{~nm}$ pitch and $\Delta=13 \mathrm{~nm}$. As $d$ was increased, the transmission dip grew larger in magnitude and shifted to larger wavelengths. The decreased transmission is attributable to an increase in Ag coverage. The shift of the minimum transmission wavelength can be understood qualitatively based on the increase in $d$ (from 665 to $320 \mathrm{~nm}$ ) and in $a$ (from 8 to 16), which are both known to cause a redshift of the surface plasmon resonance in the plane of these arrays. ${ }^{13}$

The PL enhancement spectra (Fig. 3) exhibited a redshift with increasing $d$ and $a$ that is similar to the trend in transmission dips, establishing a strong correlation between the PL enhancement and the surface plasmon resonance. This correspondence is illustrated for 187 different samples in Fig. 4(a), in which the wavelength of minimum transmission is correlated with the maximum PL enhancement wavelength. The observed trend is well fitted by a straight line with a slope of $0.97 \pm 0.03$ and a $y$ intercept of $14 \pm 17 \mathrm{~nm}$. Despite variations in $\Delta, p$, and $d$, the strong spectral correspondence between Si QD PL enhancement and particle aro AIP license or copyright, see http://apl.aip.org/apl/copyright.jsp 


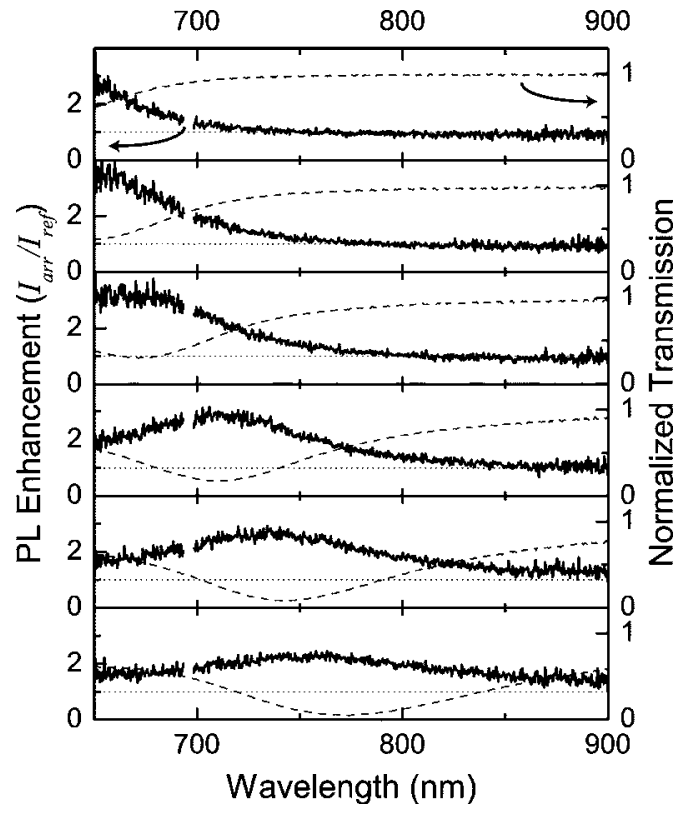

FIG. 3. PL intensity enhancement (solid lines) and normalized transmission spectra (dashed lines) for samples with $p=400 \mathrm{~nm}$ and $\Delta=13 \mathrm{~nm} ; d$ increases from the top to bottom panels, with $d=165,185,190,230,260$, and $320 \mathrm{~nm}$, respectively.

ray plasmon resonance indicates that the PL enhancement can consistently be ascribed to an increase in radiative decay rate due to resonant coupling of the Si QDs with collective surface plasmon modes of the $\mathrm{Ag}$ islands. No luminescence enhancements were observed for samples that show transmission dips resonant with the $532 \mathrm{~nm}$ excitation source, demonstrating that the increased PL intensity cannot be attributed to increased pump power absorption.

Figure 4(b) shows the magnitude of the maximum PL intensity enhancement as a function of wavelength for 201 different samples. Coupling Si QDs to Ag island arrays produces up to sevenfold enhancements, and the greatest increases are observed at short emission wavelengths, where smaller nanoparticles are resonant. It is expected that only $\mathrm{Si}$

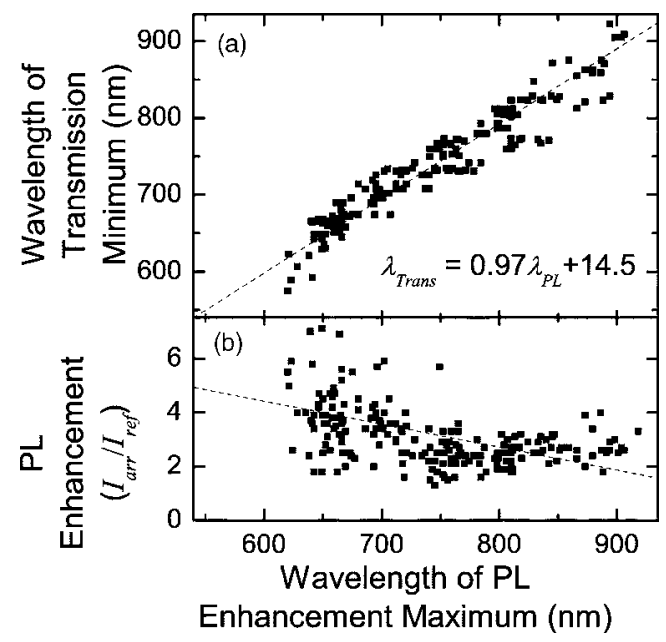

FIG. 4. (a) Correspondence between the wavelength of minimum transmission and the wavelength of maximum PL enhancement for 187 different samples. (b) Comparison between the maximum magnitude of PL enhancement and the wavelength at which this maximum is reached for 201 different samples.
QDs positioned closer to $\mathrm{Ag}$ particles than the typical plasmon coupling range of $\sim 50 \mathrm{~nm}$ experience an enhanced emission rate. ${ }^{9}$ It follows that the number of Si QDs coupled to $\mathrm{Ag}$ islands increases with particle coverage. The fact that a smaller overall PL enhancement is found for larger particles thus implies that the emission enhancement per QD must be greater near the smaller particles. This behavior is consistent with trends in radiative damping of the excited plasmon mode and in local field enhancement as a function of particle size; the former, which limits the radiative decay rate enhancement, is more significant for larger nanoparticles, ${ }^{14}$ while the local field enhancement about metal particles decreases with increasing size. ${ }^{15}$ The samples considered in Fig. 4 have $d=100-440 \mathrm{~nm}, \quad p=200-500 \mathrm{~nm}$, and $\Delta$ $=8-18 \mathrm{~nm}$, yet the magnitude of enhancement was found to depend most strongly on $d$. This indicates that the pitch has a more subtle influence, and that effects due to the $\mathrm{Si}-\mathrm{Ag}$ separation distances were likely obfuscated by the large spread in Si QD depths about the average depth $\Delta$.

In conclusion, we have observed up to sevenfold enhancements in PL intensity of Si QDs upon coupling to Ag island arrays. The enhancement can be spectrally controlled between 600 and $900 \mathrm{~nm}$ by tuning the surface plasmon resonance frequency of the Ag islands through varying the island diameter and the array pitch. Coupling to engineered plasmonic structures thus provides an interesting approach to controlling the emission rate and emission spectrum of $\mathrm{Si}$ quantum dots.

Work at AMOLF is part of the research program of FOM, supported by NOW and NANONED, a nanotechnology program of the Dutch Ministry of Economic Affairs. Sample fabrication and characterization were done at the Amsterdam nanoCenter. This work was also partially supported by NSF Grant No. CHE-0213589 and by AFOSR MURI Award No. FA9550-04-1-0434. One of the authors (H.A.A.) acknowledges the support of a Joop Los Fellowship awarded by AMOLF.

${ }^{1}$ L. T. Canham, Appl. Phys. Lett. 57, 1046 (1990).

${ }^{2}$ A. G. Cullis and L. T. Canham, Nature (London) 353, 335 (1991).

${ }^{3}$ T. Fischer, V. Petrova-Koch, K. Shcheglov, M. S. Brandt, and F. Koch, Thin Solid Films 276, 100 (1996).

${ }^{4}$ M. V. Wolkin, J. Jorne, P. M. Fauchet, G. Allan, and C. Delerue, Phys. Rev. Lett. 82, 197 (1999).

${ }^{5}$ O. Kulakovich, N. Strekal, A. Yaroshevich, S. Maskevich, S. Gaponenko, I. Nabiev, U. Woggon, and M. Artemyev, Nano Lett. 2, 1449 (2002).

${ }^{6}$ K. T. Shimizu, W. K. Woo, B. R. Fisher, H. J. Eisler, and M. G. Bawendi, Phys. Rev. Lett. 89, 117401 (2002).

${ }^{7}$ J. S. Biteen, D. Pacifici, N. S. Lewis, and H. A. Atwater, Nano Lett. 5, 1768 (2005).

${ }^{8}$ K. Okamoto, I. Niki, A. Shvartser, Y. Narukawa, T. Mukai, and A. Scherer, Nat. Mater. 3, 601 (2004).

${ }^{9}$ J. Gersten and A. Nitzan, J. Chem. Phys. 75, 1139 (1981).

${ }^{10}$ J. F. Ziegler, J. P. Biersack, and U. Littmark, The Stopping and Range of Ions in Solids (Pergamon, New York, 1985).

${ }^{11}$ K. S. Min, K. V. Shcheglov, C. M. Yang, H. A. Atwater, M. L. Brongersma, and A. Polman, Appl. Phys. Lett. 69, 2033 (1996).

${ }^{12}$ K. S. Min, K. V. Shcheglov, C. M. Yang, H. A. Atwater, M. L. Brongersma, and A. Polman, Appl. Phys. Lett. 68, 2511 (1996).

${ }^{13}$ Craig F. Bohren and Donald R. Huffman, Absorption and Scattering of Light by Small Particles (Wiley, New York, 1983).

${ }^{14}$ A. Wokaun, J. P. Gordon, and P. F. Liao, Phys. Rev. Lett. 48, 957 (1982).

${ }^{15}$ K. L. Kelly, E. Coronado, L. L. Zhao, and G. C. Schatz, J. Phys. Chem. B 107, 668 (2003). 